trode) von $-0,088$ Volt auf $-0,050$ Volt. Es zeigte schnelle Schwankungen um etwa $3 / 1000$ Volt. Die mittlere Stromdichte an der Anode betrug 5,35 mA (Abweichungen von diesem Wert kleiner als $3 \%$ ). Um Störungen durch leichtes' Wasser, das etwa der Anode noch anhaften könnte, sicher zu vermeiden, wurde der erste Teil des anodisch entwickelten Wasserstoffs verworfen. Dasjenige Gas, welches sich dann entwickelte, wurde in einem evakuierten Kolben aufgefangen und nach der Mikrowärmeleitfähigkeitsmethode von $\mathrm{F}_{\text {a }} \mathrm{ka} \mathrm{s}^{\mathbf{9}}$ analysiert. Der Aufbau der Analyseapparatur erfolgte im allgemeinen nach den Angaben von $\mathrm{F}$ a r ka s ${ }^{\mathbf{9}}$ und von $\mathrm{W}$ i r t z ${ }^{\mathbf{1 0}}$. Es wurde eine Meßgenauigkeit von etwa $0,8 \%$ erreicht.

Für die Analyse des entwickelten Gases wurden zwei Messungen ausgeführt, die beide Widerstandswerte lieferten, welche innerhalb der angegebenen Grenze unter dem Widerstandswerte für reinen leichten Wasserstoff lagen. An der Anode wurde also nur leichter Wasserstoff entwickelt. Dieser Befund beweist, daß das entwickelte Gas ausschließlich aus dem

9 A. F a k a s, Z. physik. Chem., Abt. B 22, 344 [1933]; A. u. L. F a r k a s, Proc. Roy. Soc. [London], Ser. A 144, 467 [1934]. [1936].

10 K. Wirtz, Z. physik. Chem., Abt. B 32, 334

${ }_{11} \mathrm{H}$. W i e l a n d u. H. W i n g l e r, Liebigs Ann. Chem. 431, 301 [1923].
Formaldehyd oder aus den CH-Bindungen irgendwelcher Reaktionsprodukte dieser Substanz (etwa aus Dioxymethylperoxyd, wie es sich bei der Reaktion zwischen Hydroperoxyd und Formaldehyd bildet ${ }^{\mathbf{1 1}}$ ), sicher aber nicht aus den Wassermolekülen oder den Hydroxylionen stammt.

Über den Mechanismus der Reaktion des Formaldehyds, die zur anodischen Wasserstoffentwicklung führt, existiert eine Theorie von $\mathrm{H}$ i c k l i n $\mathrm{g}^{12}$. Die Isotopenzusammensetzung des anodisch entwickelten Wasserstoffs ist mit der Hicklingschen Auffassung vereinbar, daß sich an der Anode intermediär Hydroperoxyd bildet, was sich aus dem Vergleich des hier erhaltenen Resultats mit den Ergebnissen von Wirtz und B o nh o eff e $\mathrm{r}^{7}$ über die Reaktion zwischen Formaldehyd und Hydroperoxyd ergibt.

Hrn. Prof. B on h o f fer danke ich für sein Interesse an dieser Arbeit, Frl. S. Nens und Frl. M. P a t z a k für ihre Hilfe beim Experimentieren.

12 Da Erich M ül l e r bei einem Erklärungsversuch fünfwertigen Kohlenstoff benutzt, was den gesamten Erfahrungen der organischen Chemie widerspricht, braucht auf seine Vorstellungen nicht eingegangen zu werden.

\title{
Neuere Messungen der katalytischen Spaltung des Äthylchlorids zur Prüfung des Zusammenhanges zwischen Aktivierungswärme und Aktivität
}

\author{
Von Erika Cremer und Rudolf Baldt \\ Aus dem Physikalisch-chemischen Institut der Universität Innsbruck \\ (Z. Naturforschg. 4 a, 337-341 [1949]; eingegangen am 22. November 1948)
}

1. Es werden neuere Messungen der katalytischen Spaltung des Äthylchlorids mitgeteilt an den Katalysatoren $\mathrm{PbCl}_{2}, \mathrm{BaCl}_{2}, \mathrm{CoCl}_{2}, \mathrm{NiCl}_{2}, \mathrm{CaCl}_{2}$ und $\mathrm{CdCl}_{2}$.

2. Die Katalysatoren wurden bei verschiedenen Temperaturen zwischen $600^{\circ} \mathrm{K}$ und $900^{\circ} \mathrm{K}$ vorbehandelt, wobei sich vielfach ein linearer Anstieg der Aktivierungswärme mit dieser Vorbehandlungstemperatur zeigte.

3. An $\mathrm{CaCl}_{2}, \mathrm{PbCl}_{2}, \mathrm{BaCl}_{2}$ und $\mathrm{CdCl}_{2}$ wurde die Abhängigkeit der Umsatzgeschwindigkeit vom Druck des Äthylchlorids geprüft und für den anfänglichen Umsatz $(<50 \%)$ eine Reaktion nach 1. Ordnung gefunden.

4. Es werden ferner einige Messungen über die Sedimentierungsgeschwindigkeit der untersuchten $\mathrm{BaCl}_{2}$ - und $\mathrm{NiCl}_{2}$ - Katalysatoren mitgeteilt.

5. Die gefundenen Aktivierungswärmen bewegen sich zwischen 12 und $42 \mathrm{kcal} / \mathrm{Mol}$. In dem ganzen Intervall gilt die bereits früher aufgezeigte Gesetzmäßigkeit zwischen Aktivierungswärme und Aktivität.

$\mathrm{E}$ s wurden bereits an anderer Stelle ${ }^{1}$ Ergebnisse mitgeteilt, die bei der Untersuchung der Spaltung von Athylchlorid an Chloriden erhalten wor-

1 E. Cr e m e r, Experientia 4, 9, 349 [1948]; Colloque sur la Liaison Chimique, Paris 1948, vgl. auch Österr. Chemiker-Ztg. 49, 1 [1948]. den waren und zu einer besonderen Vorstellung über den Mechanismus von heterogenen Umwandlungen an Kontakten geführt hatten. In dieser Arbeit soll nun unter Verzicht auf die theoretische Behandlung nur eine ausführliche Darstellung der experimentellen Tatsachen gegeben werden. 
Es wurde schon früher ${ }^{2}$ gezeigt, daß sich bei der Spaltung von Athylchlorid an verschiedenen Chloriden, die von $\mathrm{Sabatier}$ und $\mathrm{Mailhe}{ }^{3}$ gefunden und von Grimm und Schwambe r g e $r^{4}$ zu systematischen katalytischen Untersuchungen herangezogen worden war, eine expo- nentielle Abhängigkeit zwischen der Aktivität $A$ und der Aktivierungswärme $q$ der Arrheniusschen Gleichung

$$
k=A e^{-q / R T}
$$

ergibt. Es war dabei von besonderem Interesse, Veränderungen von $q$ und $A$ mit der Vorbehandlungstemperatur $T_{\mathrm{v}}$ festzustellen ${ }^{5}$.

Die Aufgabe dieser Arbeit war also die Messung der Umsatzgeschwindigkeit bei verschiedenen Temperaturen $T$ an Katalysatoren, die bei verschiedenen Temperaturer $T_{\mathbf{v}}$ vorbehandelt waren, ferner die Feststellung der Ordnung der Reaktion und die Auswertung der Ergebnisse nach Gl. (1). Es sei bereits hier bemerkt, daß die Reaktion nach 1 . Ordnung verläuft. Die angegebenen Aktivierungswärmen sind daher scheinbare Aktivierungswärmen, d. h. sie enthalten stets noch die Adsorptionswärme als substratives Glied.

\section{Beschreibung der Experimente}

\section{A. A p paratur}

Die Messungen wurden nach der statischen Methode vorgenommen, der Verlauf der Reaktion an Hand der Druckzunahme verfolgt. Die Apparatur bestand aus einem Reaktionsrohr aus gewöhnlichem Glas von etwa $55 \mathrm{~cm}$ Länge und etwa $5 \mathrm{~cm}$ Durchmesser. An einer Seite war (mit Schliff verbunden) ein Quecksilbermanometer für Drucke bis zu 1 at angeschlossen, auf der anderen Seite das Vorratsgefäß mit Äthylchlorid und die Leitung zur Pumpe. Das Reaktionsrohr befand sich in einem elektrischen Widerstandsofen, dessen Temperatur auf $\pm 1,5^{\circ}$ konstant gehalten wurde. Die Messung der Temperatur erfolgte mit einem Thermoelement, das zwischen Ofen und Reaktionsrohr eingeschoben war.

B. Vorbehandlung der Katalysatoren und D u r ch führung der Messungen

Die analysenreinen Substanzen wurden getrocknet und in einer Achatschale fein zerrieben. Von den so vorbereiteten Salzen wurden je $10,0 \mathrm{~g}$ in das Reaktionsrohr ohne Trägersubstanz eingewogen und über die

2 E. Cremer, Z. physik. Chem., Abt. A 144, 231 [1929].

3 Sabatier u. Mailhe, C. R. hebd. Séances Acad. Sci. 141, 238.

4 G r i m m u. S c h w a m be r g e r, Réunion intern. Chim. Phys. 3, 214 [1928]. ganze Länge hin gleichmäßig verteilt. Hierauf wurde nochmals unter Abpumpen 1 Stde. lang getrocknet und auf die unterste Vorbehandlungstemperatur $T_{\mathrm{V}}$ erhitzt. Bis $773^{\circ} \mathrm{K}$ wurde $T_{\nabla}$ um je $50^{\circ}$ variiert und der gleiche Katalysator im Ręaktionsrohr belassen. Vorbehandelt wurde jeweils 1 Stde. im Vakuum; die Genauigkeit in der Temperatur war dabei $\pm 1,5^{\circ}$. Cher $773^{\circ} \mathrm{K}$ mußte wegen Erweichung des Glases des Reaktionsrohres die Vorbehandlung in einem Muffelofen durchgeführt werden; die Genauigkeit betrug dabei nur etwa $\pm 10^{\circ}$. Außerdem mußte dann jeweils eine neue Einwaage gemacht werden.

Nach Einstellen der Versuchstemperatur $T$ wurde Athylchlorid aus dem Vorratsgefäß bis zum gewünschten Anfangsdruck in das Reaktionsgefäß eingelassen und die zeitliche Änderung des Druckes verfolgt ${ }^{6}$.

\section{Versuchsergebnisse}

\section{A. Bestimmung der Reaktionsordnung}

Zur Feststellung der Reaktionsordnung wurde die Reaktion an $\mathrm{CaCl}_{2}, \mathrm{PbCl}_{2}$ und $\mathrm{BaCl}_{2}$ über einen großen. Umsatz hin verfolgt und die Geschwindigkeitskonstanten auf ihre Konstanz ge-

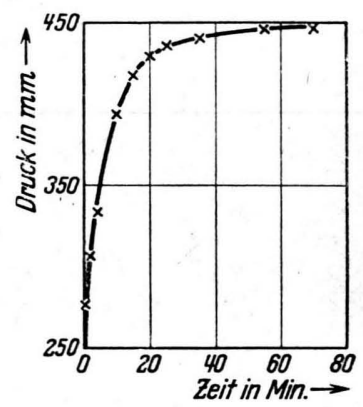

Abb. 1. Druckerhöhung bei der Äthylchloridspaltung an $\mathrm{CaCl}_{2} . T=546^{\circ} \mathrm{K}$.

prüft. Die Reaktion verläuft nach der Bruttoggleichung

$$
\mathrm{C}_{2} \mathrm{H}_{5} \mathrm{Cl}=\mathrm{C}_{2} \mathrm{H}_{4}+\mathrm{HCl} \text {, }
$$

wobei das Gleichgewicht bei den Versuchstemperaturen schon ziemlich weit nach links verschoben ist. Es wird daher der theoretische Druckanstieg auf das Doppelte, wie er bei vollständiger Spaltung zu erwarten wäre, nicht erreicht (Abb. 1). Die Geschwindigkeitskonstanten müssen dementsprechend bei weitgehendem Umsatz absinken,

5 E. Cremer u. G.-M. S chwab, Z. physik. Chem., Abt. A 144, 243 [1929]. Bezüglich dieser Mitt. und der Arbeit von G.-N. S ch w a b, Z. physik. Chem., Abt. B 5, 406 [1929], vgl. die Mitt. von E. Cremer u. R. B ald t, Mh. Chemie 49, 439 [1948].

${ }^{6}$ Die vollständige Aufführung der Versuchsdaten ist in R. B a ld t, Diss. Univ. Innsbruck 1947, enthalten. 
weil sich die Gegenreaktion bemerkbar macht, und es zeigt sich auch, daß die nach erster Ordnung berechneten Konstanten nur etwa bis zum halben Umsatz gut konstant sind.

Zur weiteren Prüfung der Reaktionsordnung wurde der Umsatz noch an denjenigen Substanzen, die die maximalen $\left(\mathrm{BaCl}_{2}\right)$ und minimalen $\left(\mathrm{CdCl}_{2}\right)$ Aktivierungswärmen ergaben, bei verschiedenen Anfangsdrucken $\left(p_{0}\right)$ gemessen. Es ergab sich dabei eine Bestätigung der 1 . Ordnung. Zum Beispiel an

$\mathrm{BaCl}_{2}\left(T=T_{\mathrm{v}}=623^{\circ} \mathrm{K}\right)$ und $\mathrm{CdCl}_{2}\left(T=T_{\mathrm{v}}=573^{\circ} \mathrm{K}\right)$

$p_{0}=504 \quad 272 \quad 93,6 \quad 277 \quad 97,0$ Torr

$k_{1}=0,40 \quad 0,42 \quad 0,43 \quad 0,28 \quad 0,29 \cdot 10^{-2} \min ^{-1}$.

B. Meßergebnisse an verschiedenen Chloriden

1. $\mathrm{PbCl}_{2}$. Bei der Spaltung an $\mathrm{PbCl}_{2}$, die zuerst untersucht wurde, zeigte (Abb. 2, Meß- gen, wie dies auch bereits von Grimm und $\mathrm{Schw}$ a mberger ${ }^{4}$ gefunden worden war. Bei $\mathrm{PbCl}_{2}$ fanden wir z. B. eine Anderung von $30 \%$ in der Aktivität bei verschiedenen Einwaagen desselben Präparats.

Eine Zusammenstellung der an Bleichlorid gefundenen $q$ - und $A$-Werte zeigt Tab. 1 .

\begin{tabular}{|c|c|c|}
\hline$T_{\mathrm{v}}\left({ }^{0} \mathrm{~K}\right)$ & $\begin{array}{c}q \\
(\mathrm{kcal} / \mathrm{Mol})\end{array}$ & $\begin{array}{c}A\left(\mathrm{~min}^{-1}\right. \\
\text { pro } 10 \mathrm{~g})\end{array}$ \\
\hline 634 & 25,1 & $5,7 \cdot 10^{5}$ \\
673 & 20,7 & $1,7 \cdot 10^{4}$ \\
713 & 31,2 & $3,7 \cdot 10^{7}$ \\
G. u. S. ${ }^{4}$ & 25,2 & \\
\hline
\end{tabular}

Tab. 1. Messungen an Bleichlorid.

Zum Vergleich ist der von Grimm und Schwamberger gemessene Wert (G.u.S.) für die Aktivierungswärme aufgeführt. Bei diesen Autoren ist

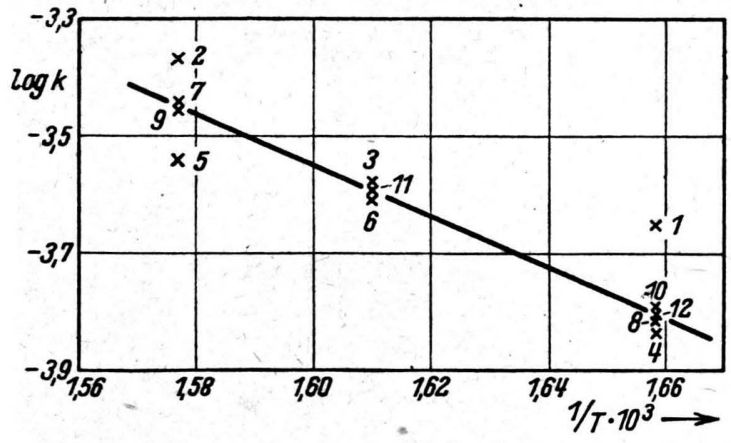

Abb. 2. Äthylchloridspaltung an $\mathrm{PbCl}_{2}$ $(\log k$ gegen $1 / T$, sämtliche Melspunkte).

punkte $1-5)^{\cdot}$ die Geschwindigkeitskonstante $\mathrm{zu}$ Beginn Schwankungen bis zu 50\%; sie wurde jedoch bei längerer Versuchsdauer (nach etwa 2 Stdn.) ziemlich gut reproduzierbar (Abb. 2, Meßpunkte 6-12). Es wurde daher bei allen fólgenden Versuchen mit den Messungen erst begonnen, nachdem die Reaktion bereits längere Zeit lief und eine starke Änderung der Aktivität nicht mehr zu erwarten war.

Bei den in den folgenden $\log k$-1/T-Diagrammen eingezeichneten Punkten ist, sofern es sich um Mittelwerte mehrerer Messungen handelt, die maximale Streubreite eingezeichnet.

Bei Präparaten gleicher Vorbehandlungstemperatur ließen sich die Aktivierungswärmen gut reproduzieren. Die Aktivitäten zeigten von Einwaage zu Einwaage ziemlich starke Schwankun-

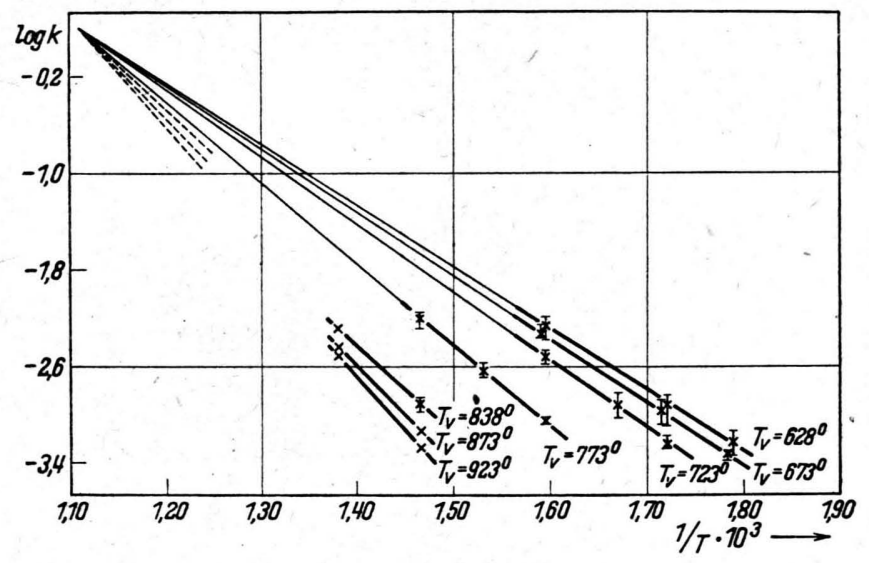

Abb. 3. Äthylchloridspaltung an $\mathrm{BaCl}_{2}(\log k$ gegen 1/T, Präparat bei verschiedenen Temperaturen vorbehandelt).

jedoch keine Vorbehandlungstemperatur angegeben. Diese wurde auch von Grimm und Schwamberger nicht systematisch variiert.

Nimmt man an, daß auf einer Oberfläche nebeneinander Zentren verschiedener katalytischer Wirksamkeit vorhanden sind, so wäre der anfängliche Anstieg der Wirksamkeit (verbunden mit einer Erniedrigung der Aktivierungswärme) durch das Freilegen besserer Zentren bei höherer Vorbehandlungstemperatur, der spätere Abfall (Ansteigen der Aktivierungswärme) durch den Abbau der jeweils aktivsten Zentren zu erklären (vgl. jedoch die Ergebnisse an $\mathrm{CdCl}_{2}$ ).

2. $\mathrm{BaCl}_{2}$. Die Reaktion am $\mathrm{BaCl}_{2}$ konnte über ein großes Intervall von $T_{\mathrm{v}}$ verfolgt werden (Abb. 3). Für $T_{\nabla}>773^{\circ} \mathrm{K}$ mußte die Vorbehand- 
lung im Muffelofen vorgenommen und daher jeweils eine neue Einwaage gemacht werden. Nach den Erfahrungen beim $\mathrm{PbCl}_{2}$ variiert dabei das $A$, nicht aber das $q$. Eine Parallelverschiebung der Kurven, wie sie durch die gestrichelten Linien angedeutet ist, läge innerhalb der Streuung bei verschiedenen Einwaagen. Im übrigen wäre diese Verschiebung der Aktivitäten minimal, da es sich höchstens um einen Faktor 2 handeln würde.

\begin{tabular}{|c|c|c|}
\hline$T_{\mathbf{v}}\left({ }^{0} \mathrm{~K}\right)$ & $q(\mathrm{kcal} / \mathrm{Mol})$ & \multicolumn{1}{|c|}{$\boldsymbol{A}$} \\
\hline 628 & 22,5 & $3,74 \cdot 10^{5}$ \\
673 & 23,0 & $4,73 \cdot 10^{5}$ \\
723 & 24,8 & $1,41 \cdot 10^{6}$ \\
773 & 30,8 & $2,58 \cdot 10^{7}$ \\
838 & 34,9 & $1,85 \cdot 10^{9}\left(3,31 \cdot 10^{9}\right)$ \\
873 & 38,2 & $1,27 \cdot 10^{9}\left(2,11 \cdot 10^{9}\right)$ \\
923 & 41,9 & $1,38 \cdot 10^{10}\left(1,95 \cdot 10^{10}\right)$ \\
\hline
\end{tabular}

Tab. 2. Messungen an Bariumchlorid.

Die eingeklammerten $A$-Werte gelten für die gestrichelten Kurven. Die Abhängigkeit der Aktivierungswärme von der Vorbehandlungstemperatur ist hier im Bereich von $673^{\circ} \mathrm{K}$ bis $923^{\circ} \mathrm{K}$ linear. Die Tatsache, daß sich alle Geraden annähernd in einem Punkte schneiden, zeigt sehr augenfällig das Bestehen der exponentiellen Beziehung zwischen $A$ und $q$ (vgl. Creme ${ }^{2}$ ).

3. $\mathrm{CoCl}_{2}$. Auch bei der Spaltung an $\mathrm{CoCl}_{2}$ zeigt sich eine allmähliche Verschlechterung der Zen- tren mit steigender Vorbehandlungstemperatur. Die Beziehung zwischen $T_{\mathrm{v}}$ und $q$ ist wie beim $\mathrm{BaCl}_{2}$ linear.

\begin{tabular}{|c|c|c|}
\hline$T_{\mathbf{v}}\left({ }^{0} \mathrm{~K}\right)$ & $q(\mathrm{kcal} / \mathrm{Mol})$ & $A$ \\
\hline 603 & 17,4 & $6,77 \cdot 10^{4}$ \\
673 & 19,2 & $2,52 \cdot 10^{5}$ \\
723 & 20,0 & $4,66 \cdot 10^{5}$ \\
773 & 22,4 & $2,93 \cdot 10^{6}$ \\
873 & 30,0 & $1,35 \cdot 10^{7}$ \\
\hline
\end{tabular}

Tab. 3. Messungen an Kobaltchlorid.

4. $\mathrm{NiCl}_{2}$. Vollkommen anders als die bisher mitgeteilten Ergebnisse fallen jene des Zerfalls an $\mathrm{NiCl}_{2}$ aus (Abb.4). Die Werte für die Aktivierungswärmen sind im gesamten Bereich zwischen $T_{\mathrm{v}}=583-873^{\circ} \mathrm{K}$ (mit einer einzigen Ausnahme bei $T_{\mathrm{v}}=723^{\circ} \mathrm{K}$ ) vollkommen konstant. Die aktiven Zentren am Nickelchlorid scheinen äußerst stabil zu sein. Nur in einem kleinen Temperatur- intervall $\left(720-750^{\circ} \mathrm{K}^{7}\right)$ bilden sich vorübergehend besonders gute Zentren aus.

5. $\mathrm{CaCl}_{2}$. Bei der Benützung von $\mathrm{CaCl}_{2}$ als Katalysator erhält man ein Bild, das im Bereich von $573-673^{\circ} \mathrm{K}$ und $773-873^{\circ} \mathrm{K}$ dem Verhalten

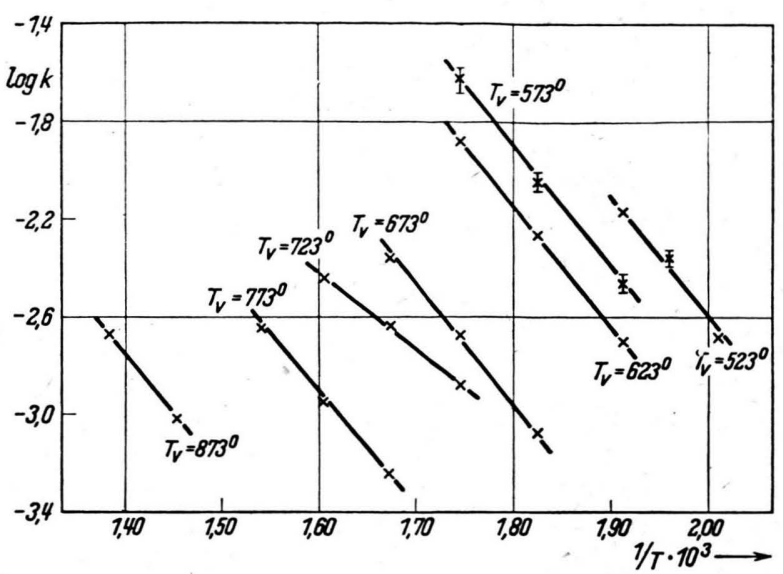

Abb. 4, Äthylchloridspaltung an $\mathrm{NiCl}_{2}$ ( $\log k$ gegen $1 / T$, Präparat bei verschiedenen Temperaturen vorbehandelt).

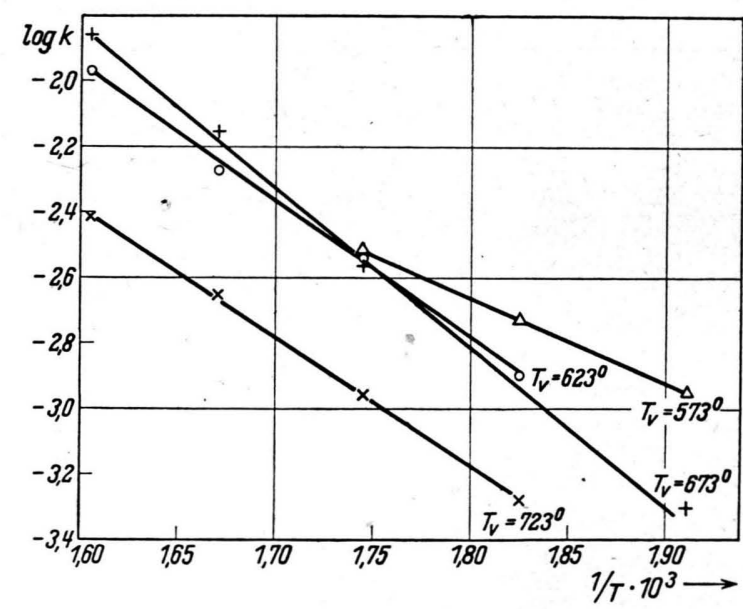

Abb. 5. Äthylchloridspaltung an $\mathrm{CdCl}_{2}$ ( $\log k$ gegen 1/T, Präparat bei verschiedenen Temperaturen vorbehandelt).

der Blei-, Barium- und Kobaltsalze entspricht, in dem dazwischen liegenden Intervall aber dem $\mathrm{NiCl}_{2}$.

6. $\mathrm{CdCl}_{2}$. Wie aus Abb. 5 zu ersehen ist, wächst beim $\mathrm{CdCl}_{2}$ bei steigender Vorbehandlungstemperatur zunächst die Aktivierungswärme wie bei den

7 Bei dieser Temperatur findet eine Farbänderung von hellgelb in dunkelgoldgelb statt. Außerdem beginnt hier, bei Erhitzung an der Luft, die Abgabe von Chlor und der Čbergang in Nickeloxyd (unveröff. Messungen von $\mathrm{H}$. S chne id e r). 
Substanzen $1-3$. Bei $T_{\mathrm{v}}=723^{\circ} \mathrm{K}$ treten ähnlich wie bei dieser Temperatur am Nickelchlorid wieder Zentren mit niedrigerem $q$ auf.

\begin{tabular}{|c|c|c|}
\hline$T_{\nabla}\left({ }^{0} \mathrm{~K}\right)$ & $q(\mathrm{kcal} / \mathrm{Mol})$ & $A$ \\
\hline 573 & 14,0 & $2,87 \cdot 10^{4}$ \\
623 & 16,6 & $2,27 \cdot 10^{5}$ \\
673 & 19,3 & $2,29 \cdot 10^{3}$ \\
723 & 19,3 & $1,72 \cdot 10^{6}$ \\
773 & 19,3 & $3,99 \cdot 10^{5}$ \\
873 & 26,2 & $3,50 \cdot 10^{5}$ \\
G. u.S. & 18,6 & \\
\hline
\end{tabular}

Tab. 4. Messungen an Calciumchlorid.

Die unter 1 besprochene Möglichkeit anfänglich nebeneinander bestehender Zentren ist hier auszuschließen. Es müßte sonst die $623^{\circ}$-Kurve oberhalb des Schnittpunktes mit der $673^{\circ}$ - Kurve in diese übergehen. Dies ist aber nicht der Fall, wovon wir uns

\begin{tabular}{|c|c|c|}
\hline$T_{\mathrm{\nabla}}\left({ }^{0} \mathrm{~K}\right)$ & $q(\mathrm{kcal} / \mathrm{Mol})$ & $A$ \\
\hline$\tilde{5} 73$ & 11,6 & 70 \\
623 & 18,1 & $2,28 \cdot 10^{4}$ \\
673 & 22,1 & $7,95 \cdot 10^{5}$ \\
723 & 19,3 & $2,53 \cdot 10^{4}$ \\
\hline
\end{tabular}

Tab. 5. Messungen an Cadmiumchlorid.

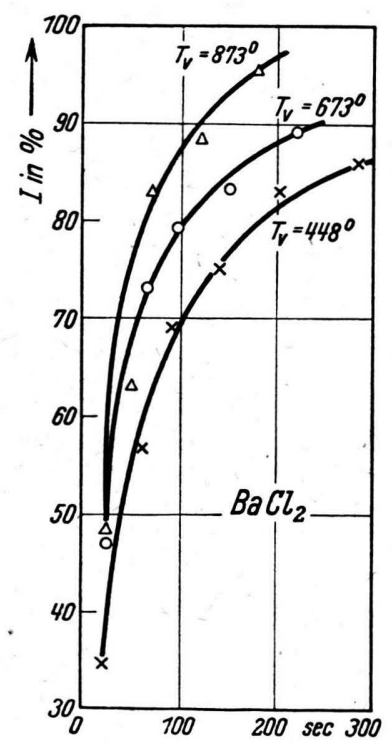

Abb. 6. Zeitliche Abhängigkeit der Trübung einer Aufschwemmung von $\mathrm{BaCl}_{2}$ in Cyclohexan. Die 3 Kurven entsprechen drei verschiedenen Vorbehandlungstemperaturen. nochmals durch Wiederholung der Messungen überzeugten.

Bei $T_{\boldsymbol{v}}=773^{\circ} \mathrm{K}$ war die Aktivität so stark gesunken, daß die Reaktion nicht mehr gemessen werden konnte.

C. Feststellung der Të̈lchengrößenveränderung mit der Vorbehandlungstem peratur

Um weitere Anhaltspunkte für die Veränderung der Teilchengröße und damit der Oberfläche bei den verschiedenen Katalysatoren $\mathrm{zu}$ erhalten, wurden auch Sedimentierungsversuche durchgeführt. Es ergab sich, daß die höher erhitzten Pulver stets schneller sedimentierten. Besonders groß war der Unterschied beim Nickelchlorid, verhältnismäßig gering beim Bariumchlorid, in guter Übereinstimmung mit dem katalytischen Befund. Die Abb. 6 u. 7 zeigen Kurven, bei denen als Maß für die durch die Aufschlämmung eines Pulvers in Cyclohexan entstandene Trübung die mittels eines Pulfrichschen Stufenphotometers gemessene Schwächung des durchgehenden Lichtes benützt wurde. Es ist in den Abbildungen die durchgehende Lichtmenge (in \% der einfallenden Menge) gegen die Zeit aufgetragen.

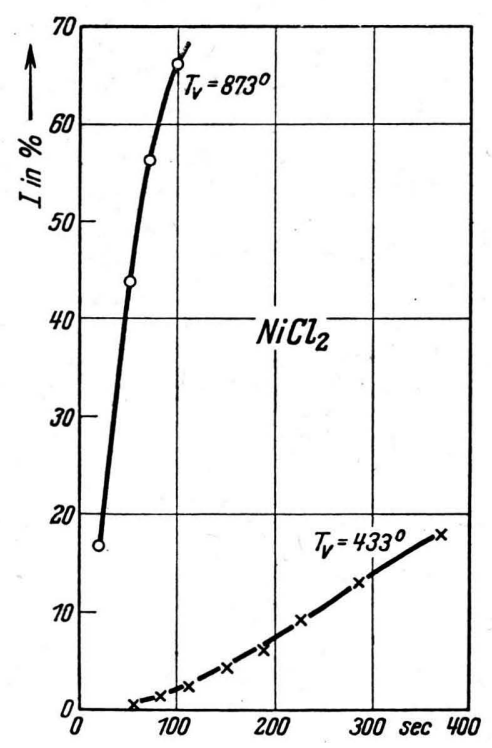

Abb. 7. Zeitliche Abhängigkeit der Trübung einer Aufschwemmung von $\mathrm{BaCl}_{2}$ in Cyclohexan. Die beiden Kurven entsprechen zwei verschiedenen Vorbehandlungstemperaturen. 